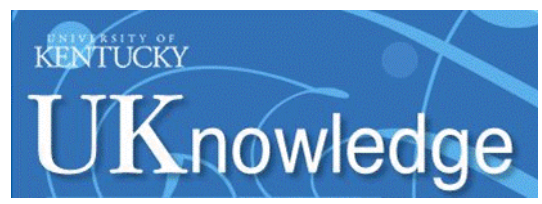

University of Kentucky

UKnowledge

$11-22-2016$

\title{
Psychological Needs, Self-Regulation, and Motivation Profiles Among a Sample of Hong Kong Chinese University Students: A Person-Centered Approach
}

\author{
Cecilia M. S. Ma \\ The Hong Kong Polytechnic University, China \\ Daniel T. L. Shek \\ University of Kentucky \\ Catie C. W. Lai \\ The Hong Kong Polytechnic University, China
}

Follow this and additional works at: https://uknowledge.uky.edu/pediatrics_facpub

Part of the Chinese Studies Commons, Higher Education Commons, Mental and Social Health Commons, and the Psychology Commons

Right click to open a feedback form in a new tab to let us know how this document benefits you.

\section{Repository Citation}

Ma, Cecilia M. S.; Shek, Daniel T. L.; and Lai, Catie C. W., "Psychological Needs, Self-Regulation, and Motivation Profiles Among a Sample of Hong Kong Chinese University Students: A Person-Centered Approach" (2016). Pediatrics Faculty Publications. 283.

https://uknowledge.uky.edu/pediatrics_facpub/283

This Article is brought to you for free and open access by the Pediatrics at UKnowledge. It has been accepted for inclusion in Pediatrics Faculty Publications by an authorized administrator of UKnowledge. For more information, please contact UKnowledge@lsv.uky.edu. 


\section{Psychological Needs, Self-Regulation, and Motivation Profiles Among a Sample of Hong Kong Chinese University Students: A Person-Centered Approach}

\section{Digital Object Identifier (DOI)}

https://doi.org/10.1515/ijdhd-2017-7009

\section{Notes/Citation Information}

Published in International Journal on Disability and Human Development, v. 16, issue 4, p. 407-416.

(C2017 Walter de Gruyter GmbH, Berlin/Boston.

The copyright holder has granted the permission for posting the article here. 
Cecilia M.S. Ma*, Daniel T.L. Shek and Catie C.W. Lai

\section{Psychological needs, self-regulation, and motivation profiles among a sample of Hong Kong Chinese university students: a person-centered approach}

DOI 10.1515/ijdhd-2017-7009

Received August 5, 2016; accepted September 10, 2016; previously published online November 22, 2016

\begin{abstract}
To better understand factors that influence individuals' motivation to engage in physical activity, this study aimed to identify the motivational profiles among the Chinese university students, compare the motivational profiles between genders, and explore whether satisfaction of various psychological needs vary with different motivational profiles. Two thousand and twenty-two Chinese university students completed a battery of selfreport questionnaires assessing motivation regulation and psychological needs satisfaction. Three motivational profiles were identified for both genders. The overall patterns of the motivational profiles for both genders were similar. However, males (44\%) were more likely than their female counterparts (19\%) in self-determined profile group. Students with self-determined motivation profile, compared to non self-determined motivation profile, had higher values on three types of needs satisfaction. Findings and implication of the study were discussed.
\end{abstract}

Keywords: motivational profiles; person-centered; psychological needs; self-determination.

\footnotetext{
*Corresponding author: Cecilia M.S. Ma, Department of Applied Social Sciences, The Hong Kong Polytechnic University, Hunghom, Hong Kong, P.R. China, E-mail: cecilia.ma@polyu.edu.hk Daniel T.L. Shek: Department of Applied Social Sciences, The Hong Kong Polytechnic University, Hong Kong, P.R. China; Centre for Innovative Programmes for Adolescents and Families, The Hong Kong Polytechnic University, Hong Kong, P.R. China; Department of Social Work, East China Normal University, Shanghai, P.R. China; Kiang Wu Nursing College of Macau, Macau, P.R. China; and Division of Adolescent Medicine, Kentucky Children's Hospital, University of Kentucky, Lexington, KY, USA

Catie C.W. Lai: Department of Applied Social Sciences, The Hong Kong Polytechnic University, Hong Kong, P.R. China
}

\section{Introduction}

Physical inactivity is one of the major health risk behaviors in college students [1] and has been identified as one of the priorities in the Healthy People 2010 objectives [2] and Healthy Campus 2010 Campaign Objectives [1]. Health professionals and researchers call for the need to combat sedentary life among college students [3]. Therefore, further research on this population is warranted to better understand factors that influence individuals' motivation to engage in physical activity.

Among numerous studies on sport and exercise motivation in literature, self-determination theory $(\mathrm{SDT})[4,5]$ is one of the widely used theoretical perspectives on the relationship between motivation and physical activity [6-8]. According to SDT, motivations lie on a continuum from self-determined to non self-determined, from intrinsic, external motivations to amotivation [9]. Intrinsic motivation refers to individuals engaging in physical activity because of inherent feelings, such as pleasure, fun and satisfaction; extrinsic motivation occurs when individuals decide to do exercise, not because of inherent enjoyment [4]. Extrinsic motivation consists of four types of regulation. They are integrated, identified, introjected and external, in descending order in the motivation continuum [4]. Integrated regulation happens when participation in physical activity is to meet personal values, beliefs, and goals. Identified regulation occurs when individuals participate in physical activity because they consider the activity is valuable and important to them. Introjected regulation refers to participation in physical activity due to pressures, such as to avoid shame and guilt or to gain social recognition and feelings of worth. External regulation appears when participation in physical activity because of external demand or socially constructed contingency, such as reward, prize, or punishment. At the self-determined bottom of the continuum is amotivation, which characterizes as a lack of intrinsic or extrinsic intention to do exercise [9]. The fundamental premise of SDT is that self-determined motivation is facilitated 
by the social environment that provides the satisfaction of three basic psychological needs, including relatedness (connectedness with others), competence (efficacy to complete an optimal challenge task), and autonomy (provision of choice, pressure free, sense of volition) [9]. Conversely, the social environment, thwarting these three needs, forestalls the internalization of behavioral regulation [9]. Partial internalization occurs when the social environment provides the satisfaction of competence and relatedness as the presence of autonomy is essential for full internalization [9]. For example, they implied that the process of internalization can be forestalled, and therefore introjected regulation is fostered by the satisfaction of needs for relatedness in an environment with external pressures and controls (i.e. the absence of autonomy).

Koestner and Losier [10] supported that satisfaction of the need for autonomy is a chief element for progress in internalization. They, however, suggested different patterns of internalization by specifying the correlations between satisfaction of the three needs and introjected, identified, and intrinsic behavioral regulations. Introjected and identified behavioral regulations are nurtured by the satisfaction of the need for both autonomy and relatedness in a conflicting and harmonious environment, respectively. Instead of satisfaction of all three needs, relatedness is a less salient factor for promoting intrinsic regulation. Intrinsic regulation can occur in an environment providing support to competence and autonomy. Therefore, more research is warranted to examine the psychological needs for promoting self-determined motivation.

Research within the SDT framework has shown that higher degree of self-determined behavioral regulation is related to higher level of physical activity in cross-sectional [11, 12], longitudinal [13], and experimental studies [14]. This is further supported by Teixeira and colleagues [15], who reviewed 66 empirical SDT studies, and found that self-determined motivation (i.e. intrinsic and identified regulation) is a significant predictor of more adaptive behavioral outcomes (exercise adherence, effort, and persistence). However, prior studies were mainly conducted in western contexts without exploring gender difference on motivation.

First, there were very few studies which examined the differences in motivation in non-western cultures. Duda and colleagues $[16,17]$ noted the role of culture in influencing individuals' cognition, affect and behavioral outcomes in physical activity research. SDT posits that culture is a critical factor influencing one's internalization that influences the development of autonomous regulations (self-determined motivation) and the decision-making process [9]. The emphasis on interdependence may influence the internalization of motivation in a collectivistic society [18]. Individuals in eastern culture may be more internalized on conforming to the social norms and thus perceive such "action" as in line with their personal values. This may undermine the negative influence of the absence of autonomy, as posited in SDT [19]. The presence of autonomy is less crucial on facilitating the process of internalization and promoting self-determined motivation in collectivistic and non-western contexts [20]. Iyengar and Lepper [20] found that Asian individuals were more intrinsic motivated when the decision of participation in an activity was made by people they trusted, compared to personal choice. Given the cultural influence on internalization of motivation, it is vital to explore the relations between satisfaction of the three needs and the internalization of motivation.

Second, there are scarce studies adopting SDT framework to understand gender differences in motivation and needs satisfaction. Past studies showed that males were more likely to meet the American College of Sports Medicine guidelines of physical activity than females [21]. This is further supported in Chinese samples [22, 23]. Such gender differences may relate to the motivation to participate in physical activity. Research in education literature revealed that females displayed a more self-determined motivation and reported a lower level of dropout than their male counterparts [24]. However, very few studies assess the gender differences in motivation regulations as a function of the greater amount of physical activity. To promote students' physical health, more research is warranted to examine factors (relatedness, competence, and autonomy) that fostering internalization of behavioral regulation, so as to encourage more participation in and less dropout from physical activity.

Third, prior studies usually adopted a variablecentered approach in testing the relationships between each type of motivation and other variables. More specifically, a single indicator [i.e. self-determination index (SDI)] is widely used to assess individual's motivation to participate in certain behavior [24-26]. However, such method may fail to capture the multidimensionality of motivation and ignore the differences in the magnitude and direction of various patterns of correlations among motivation types. Moran and colleagues [27] argue that "this ignores the possibility that distinct constellations of motivational profiles exist in the population and these SDT motivation profiles may correspond to differences in other variables" (p. 354). A profile approach may provide unique information to study the relations between motivation and theoretically relevant antecedents and 
consequences. "Inconsistent support for the relationships of introjected and external regulations to theoretically relevant constructs may be due to examining regulation types in parallel rather than in combination" [28]. Wang and colleagues suggest the use of person-oriented approach, such as latent profile analysis, to show how individuals differ in their motivational profile and the unique effect of each type of behavioral regulation on theoretically relevant factors $[26,29,30]$. By using a person-centered approach, Moran et al. [27] found the self-determined and motivated groups perceived the greater satisfaction of the three needs, compared to the low introjection and low autonomy motivated groups. Therefore, more research is warranted to explore the diverse patterns of motivation in non-western contexts, possible differences in motivation profiles by gender, and possible variations in satisfaction of the psychological needs among motivation profiles.

\section{A person-oriented approach - latent profile analysis (LPA)}

Latent profile analysis (LPA), one of the person-centered approaches, assesses the latent structure of motivation and allows us to identify the homogeneous patterns of motivation groups of people who can be classified according to typologies. This method extends the traditional variable-centered research by considering intra-individual variation in motivation and capturing a better picture of an individual [31]. LPA allows us to group individuals into profiles based on homogenous characteristics and to identify differences in satisfaction of psychological needs among different motivation profiles. Such research is beneficial for understanding how each motivation profile is uniquely related to other SDT variables.

In general, three motivational profiles (high selfdetermined, moderate self-determined, and low selfdetermined/amotivated) were found in academic [32], physical education [33-35], and sports contexts [36]. However, Cox and colleagues [34] recently found a fourcluster solution (highly diverse motivated, moderate motivated, moderate diverse motivated, and externally motivated) when assessing the relationship between motivation profiles and outcomes affect (enjoyment), behavioral (effort), and cognitive (social physique anxiety) outcomes. Similarly, Moran and colleagues [27] explored the patterns of motivation and the linkages of each profile and outcomes (e.g. job performance, needs satisfaction) and found a five-cluster solution. Given the inconsistency of the motivational profiles in terms of quantity (number of profile/class membership) and quality (i.e. a combination of different types of motivation regulation), further research in this area is warranted.

The purposes of the study were threefold: (1) to identify the motivational profiles among the Chinese university students; (2) to compare the motivational profiles between male and female students; and (3) to explore whether satisfaction of various psychological needs and vary with different motivational profiles. Based on the prior literature, high levels of self-determined motivation are expected to have higher perceptions of needs satisfaction.

\section{Methods}

A total of 2022 Chinese participants (Male: $\mathrm{n}=965$, 47.7\%; Female: $\mathrm{n}=1057,52.2 \%$ ) participated in the study. The participants were on average 18.73 [standard deviation $(\mathrm{SD})=1.29$ ]. The majority of participants were Chinese $(n=1896,94 \%)$, freshmen $(n=1952,96.7 \%)$, and lived off-campus ( $n=1316,65.5 \%)$.

\section{Measures}

Participants' level of motivation toward exercise was measured by the Behavioral Regulation in Exercise Questionnaire-2 (BREQ-2) [37]. The scale comprises 19 items and measures an individual's five types of motivation, including intrinsic motivation (four items), identified motivation (four items), introjected regulation (three items), external regulation (four items), and amotivation (four items). The study followed the practice as suggested by [32], integrated regulation whose identity is still undergoing formation, is not examined in our sample. Responses were indicated on a 5-point Likert-type scale ranging from 1 (Strongly disagree) to 5 (Strongly agree). Previous work has supported the construct validity and reliability of this scale [13]. This scale has been used among college sample in Canada [38, 39], the United Kingdom [39], adults in Greece [40] and in the USA [41]. Cronbach's alphas of all subscales are satisfactory (identified regulation: 0.75; external regulation: 0.80 ; introjected regulation: 75 ; intrinsic regulation: 86 ; amotivation: 86 ).

Psychological needs satisfaction was assessed using the Psychological Needs Satisfaction in Exercise Scale (PNSES) [42]. The scale comprises 18 items measuring three subscales including autonomy (six items), competence (six items), and relatedness (six items). Responses are provided on a 6-point Likert scale, ranging from 1 (Strongly disagree) and 6 (Strongly agree). The validity and reliability of the scale have been shown in a prior study [43]. This scale has been conducted among college sample in Canada [13, 44]. Cronbach's alphas of all subscales in the present study are satisfactory (competence: 0.94; autonomy: 0.89; relatedness: 0.91).

A back-translation procedure [45] was used to measure the meaning equivalence of the instrument. The original questionnaire was developed in English and translated into Chinese by the author who is fluent in both English and Chinese. Then, without referring to the original scale, the backward translation was done 
by a professional Chinese translator, who is bilingually fluent in English and Chinese. Then, two independent researchers compared the backward translation version with the original version to ensure the content of both versions was the same. No deviation in meaning was found. The study received the approval of the University Human Research Ethics Committee. Informed consent was obtained before completing the questionnaire.

Descriptive statistics and internal consistency reliability were completed using the IBM SPSS Statistics for Window, Version 22.0 (SPSS Inc., Chicago, IL, USA). All LPA models were performed using MPlus version 7.3. Multiple criteria were used to select the best-fitting model [46, 47], including the Akaike Information Criterion (AIC), the Bayesian Information Criterion (BIC), and the adjusted-BIC (ABIC). Lower values of these indices suggest better fit of the data. Also, the Lo-Mendell-Rubin test (LMRT) and Vuong-Lo-MendellRubin (VLMRL) were employed to compare the increase in model fit between $\mathrm{k}-1$ class and k class models. Significant $\mathrm{p}$-values $(\mathrm{p}<0.05)$ indicate a target model ( $\mathrm{k}$-class solution) fits the data better than the model with one fewer class (k-1 class solution) [48]. Finally, entropy is inspected, ranging from 0 to 1 with higher values of entropy indicating the better classification of individuals [49]. Interpretability and theoretical appropriateness were also considered [31]. Age was included as an auxiliary variable to investigate whether this demographic variable influences latent class assignment. This approach allowed us to decrease bias when estimating the parameters without influencing the model itself [50]. In the present study, LPA was conducted separately for each gender group.

\section{A latent profile solution}

To perform LPA, all models were estimated in Mplus (version 7.30) by using maximum likelihood robust (MLR). Missing data were low (ranged from $0.3 \%$ to $1.6 \%$, Table 1) and were estimated using an expectation-maximization (EM) algorithm [50]. Several solutions with 1-5 classes were tested to identify the optimal number of classes. Once the optimal number of motivational profiles is identified, a oneway multivariate analysis of covariance (MANCOVA) with Bonferroni adjustment post-hoc test was used to test differences in needs satisfaction. Needs satisfaction served as dependent variables; motivational profile groups were the independent variable. Effect sizes of 0.01 is small, 0.06 is moderate, and 0.16 is high [51].

\section{Results}

All data were normally distributed (i.e. skewness $<2.0$; kurtosis <7.0) [52]. Descriptive statistics and internal consistencies can be found in Table 1. To examine the factor structure of the BREQ-2 and PNSES, confirmatory factor analysis (CFA) was performed. A model is considered acceptable with a cut-off criteria of a comparative fit index (CFI) of 0.90 or $[53,54]$, a root mean square error of approximation (RMSEA) of $0.10[55,56]$ or below and a standardized root mean square residual (SRMR) with 0.08 or less [53]. The factorial validity of the three-factor correlated structure of the PNSES is supported, $\chi^{2}(132)=2089.03$; $\mathrm{p}<0.01$, RMSEA $=0.09(90 \% \mathrm{CI}=0.08-0.09), \mathrm{CFI}=0.93$, $\mathrm{TLI}=0.92, \quad \mathrm{SRMR}=0.05$. The factor structure of the BREQ-2 showed a moderate fit of the five-factor model, $\chi^{2}(142)=2040.27, \quad \mathrm{RMSEA}=0.08 \quad(90 \% \mathrm{CI}=0.08-0.08)$, $\mathrm{CFI}=0.90, \mathrm{SRMR}=0.07$. An inspection of the modification indices suggested the error variances of the two indicators of external regulation should be related $(\mathrm{MI}=216.27)$. A modified model, therefore, was tested and showed a slightly improved fit, $\chi^{2}(141)=1845.45$, RMSEA $=0.08(90 \%$ $\mathrm{CI}=0.07-0.08), \mathrm{CFI}=0.91, \mathrm{SRMR}=0.07$. The results of the CFA show the factorial validity of the BREQ-2. Internal consistency of all subscales is satisfactory (above 0.70, Table 1).

\section{Number of class}

Male - Several models from one-class to five-class solutions were fitted (Table 2). The two-class solution was better than one-class as indicated by lower BIC, AIC, ABIC and significant VLMR, LMRT. The three-class solution was better than two-class solution due to lower values of BIC, AIC and ABIC and a higher entropy value. Compared to

Table 1: Descriptive statistics, internal consistencies and correlations of variables.

\begin{tabular}{|c|c|c|c|c|c|c|c|c|c|c|c|c|c|}
\hline & $\begin{array}{l}\text { Whole } \\
\text { M (SD) }\end{array}$ & $\begin{array}{r}\text { Male } \\
M(S D)\end{array}$ & $\begin{array}{l}\text { Female } \\
M \text { (SD) }\end{array}$ & a & 1 & 2 & 3 & 4 & 5 & 6 & 7 & 8 & 9 \\
\hline 1. External & $2.08(0.75)$ & $2.02(0.74)$ & $2.13(0.76)$ & 0.81 & - & & & & & & & & \\
\hline 2. Introjected & $2.92(0.86)$ & $2.98(0.88)$ & $2.87(0.84)$ & 0.75 & -0.02 & - & & & & & & & \\
\hline 3. Identified & $3.53(0.78)$ & $3.65(0.70)$ & $3.41(0.68)$ & 0.75 & $-0.18^{a}$ & $0.68^{a}$ & - & & & & & & \\
\hline 4. Intrinsic & $3.78(0.78)$ & $4.02(0.71)$ & $3.57(0.77)$ & 0.86 & $-0.27^{\mathrm{a}}$ & $0.35^{\mathrm{a}}$ & $0.58^{\mathrm{a}}$ & - & & & & & \\
\hline 5. Amotivation & $1.62(0.63)$ & $1.58(0.63)$ & $1.66(0.63)$ & 0.86 & $0.57^{\mathrm{a}}$ & $-0.18^{a}$ & $-0.39^{a}$ & $-0.38^{a}$ & - & & & & \\
\hline 6. Competence & $3.97(1.09)$ & $4.22(1.04)$ & $3.74(1.07)$ & 0.94 & $-0.21^{\mathrm{a}}$ & $0.31^{\mathrm{a}}$ & $0.50^{\mathrm{a}}$ & $0.61^{a}$ & $-0.27^{\mathrm{a}}$ & - & & & \\
\hline 7. Autonomy & $4.63(0.81)$ & $4.75(0.78)$ & $4.52(0.82)$ & 0.89 & $-0.29^{a}$ & $0.20^{\mathrm{a}}$ & $0.41^{\mathrm{a}}$ & $0.48^{\mathrm{a}}$ & $-0.37^{a}$ & $0.53^{\mathrm{a}}$ & - & & \\
\hline 8. Relatedness & $4.18(0.94)$ & $4.38(0.92)$ & $3.99(0.92)$ & 0.91 & $-0.16^{a}$ & $0.36^{a}$ & $0.53^{a}$ & $0.69^{a}$ & $-0.31^{a}$ & $0.71^{\mathrm{a}}$ & $0.56^{a}$ & - & \\
\hline Missing values (\%) & & & & & 0.3 & 0.4 & 0.4 & 0.3 & 0.5 & 0.4 & 0.5 & 0.6 & 1.6 \\
\hline
\end{tabular}

${ }^{\mathrm{a} p}<0.01$. 
Table 2: Results of latent profile analysis (LPA) for male.

\begin{tabular}{|c|c|c|c|c|c|c|c|}
\hline Number of class & AIC & BIC & $A B I C$ & VLMR p-value & LMR-A p-value & BLRT p-value & Entropy \\
\hline 1 & 10590.523 & 10638.832 & 10607.073 & - & - & - & - \\
\hline 2 & 9902.420 & 9979.714 & 9928.899 & 0.0000 & 0.0000 & $<0.0000$ & 0.695 \\
\hline 3 & 9466.701 & 9572.980 & 9503.111 & 0.0461 & 0.0489 & $<0.0000$ & 0.804 \\
\hline 4 & 9292.620 & 9427.885 & 9338.960 & 0.5466 & 0.5526 & $<0.0000$ & 0.854 \\
\hline 5 & 9012.086 & 9176.336 & 9068.356 & 0.0369 & 0.0400 & $<0.0000$ & 0.859 \\
\hline
\end{tabular}

AIC, Akaike Information Criterion; BIC, Bayesian Information Criterion; ABIC, Adjusted-BIC (ABIC); VLMR, Vuong-Lo-Mendell-Rubin-likelihood ratio test; LMR-A, Lo-Mendell-Rubin adjusted likelihood ratio test; BLRT, Bootstrapped likelihood ratio test.

the five-class solution, the four-class solution indicated better fit with higher entropy. Despite the lower values of BIC, AIC, and ABIC of the four-class solution, the LMRT and VLRT were not statistically significant from the threeclass solution. Also, the four-class and five-class solutions were rejected as one of the profiles is less than $2 \%$ (i.e. four-class: $1.2 \%$ of participants; five-class: $1.5 \%$ of participants). Therefore, the three-class solution was considered the best model. Entropy was 0.80 with average posterior probabilities ranged from 0.90 to 0.93 .

Female - Similar results were shown in the female group (Table 3). The three-class solution fitted the data better as compared to the two-class solution due to the lower values of AIC, BIC, and ABIC. The four-class and five-class solutions were rejected as one of the profiles with only 19 participants (less than 2\%). A three-class solution was, therefore, deemed optimal. Entropy 0.74 with class probabilities ranged from 0.87 to 0.89 .

\section{Motivational profiles}

Male - The first class was named as moderately motivated group ( $\mathrm{n}=473,51 \%$ ) (see Figure 1). This class shows high intrinsic motivation $(\mathrm{M}=3.73, \mathrm{SE}=0.05)$ and identified motivation $(M=3.27, S E=0.04)$, moderate introjected motivation $(\mathrm{M}=2.58, \mathrm{SE}=0.05)$, external regulation $(\mathrm{M}=2.24, \mathrm{SE}=0.05)$, and external regulation $(\mathrm{M}=2.24, \mathrm{SE}=0.05)$ and low amotivation $(\mathrm{M}=1.77, \mathrm{SE}=0.06)$. The second class was named as self-determined group $(\mathrm{n}=408,44 \%)$. This class shows high intrinsic motivation $(\mathrm{M}=4.46, \mathrm{SE}=0.03)$, identified motivation $(M=4.20, S E=0.05)$, and introjected $(M=3.48$, $\mathrm{SE}=0.08)$, low external regulation $(\mathrm{M}=1.65, \mathrm{SE}=0.05)$, and amotivation $(\mathrm{M}=1.20, \mathrm{SE}=0.03)$. The third class was named as non self-determined group $(n=45,5 \%)$. This class shows high amotivation $(\mathrm{M}=3.72, \mathrm{SE}=0.30)$ and moderate external regulation $(\mathrm{M}=2.96, \mathrm{SE}=0.15)$, low intrinsic motivation $(M=2.72, S E=0.25)$, identified motivation $(M=3.02$, $\mathrm{SE}=0.17)$, and introjected motivation $(\mathrm{M}=3.05, \mathrm{SE}=0.20)$. The inclusion of age as an auxiliary variable shows that age had no effect on the class assignment $(\mathrm{p}>0.05)$.

Female - the first class was named as moderately motivated group $(n=558,55 \%)$ (see Figure 2). This class shows moderate identified motivation $(M=3.43$, $\mathrm{SE}=0.09)$, intrinsic motivation $(\mathrm{M}=3.63, \mathrm{SE}=0.07)$, introjected $(M=2.94, S E=0.11)$, and external regulation $(\mathrm{M}=2.27, \mathrm{SE}=0.05)$, low amotivation $(\mathrm{M}=1.69, \mathrm{SE}=0.05)$. The second class was named as non self-determined group $(n=263,26 \%)$. This class shows moderate intrinsic motivation $(\mathrm{M}=2.88, \mathrm{SE}=0.14)$, identified motivation $(M=2.68, S E=0.08)$, external regulation $(M=2.16$, $\mathrm{SE}=0.06)$ and introjected $(\mathrm{M}=2.03, \mathrm{SE}=0.09)$, low amotivation $(M=1.99, S E=0.09)$. The third class was named as self-determined group $(n=201,19 \%)$. This class shows high identified motivation $(M=4.35, S E=0.10)$, intrinsic motivation $(M=4.31, S E=0.06)$ and introjected $(M=3.78$, $\mathrm{SE}=0.10)$, low external regulation $(\mathrm{M}=1.77, \mathrm{SE}=0.07)$, and amotivation $(\mathrm{M}=1.25, \mathrm{SE}=0.05)$.

Table 3: Results of latent profile analysis (LPA) for female.

\begin{tabular}{|c|c|c|c|c|c|c|c|}
\hline Number of class & AIC & BIC & $A B I C$ & VLMR p-value & LMR-A p-value & BLRT p-value & Entropy \\
\hline 1 & 11562.926 & 11612.222 & 11580.461 & - & - & - & - \\
\hline 2 & 10767.986 & 10846.858 & 10796.040 & $<0.0000$ & $<0.0000$ & $<0.0000$ & 0.695 \\
\hline 3 & 10485.185 & 10593.635 & 10523.760 & 0.0216 & 0.0233 & $<0.0000$ & 0.743 \\
\hline 4 & 10261.428 & 10399.455 & 10310.523 & 0.2270 & 0.2339 & $<0.0000$ & 0.798 \\
\hline 5 & 10052.685 & 10220.288 & 10112.301 & 0.3506 & 0.3563 & $<0.0000$ & 0.787 \\
\hline
\end{tabular}

AIC, Akaike Information Criterion; BIC, Bayesian Information Criterion; ABIC, Adjusted-BIC (ABIC); VLMR, Vuong-Lo-Mendell-Rubin-likelihood ratio test; LMR-A, Lo-Mendell-Rubin adjusted likelihood ratio test; BLRT, Bootstrapped likelihood ratio test. 


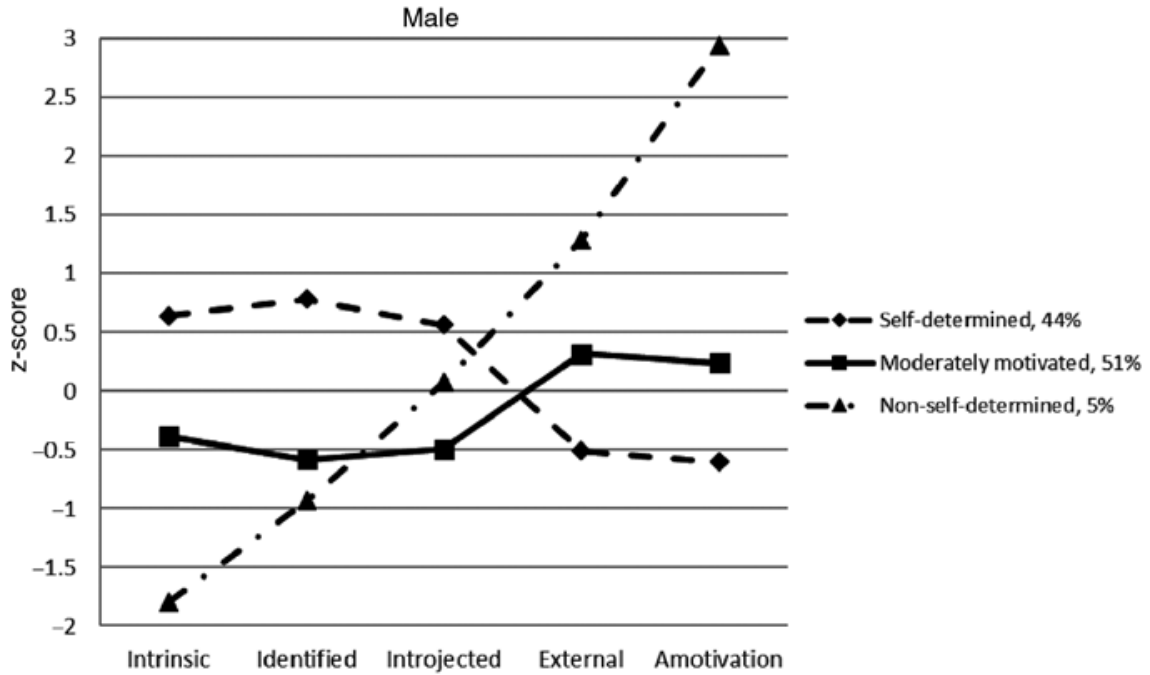

Figure 1: Profile characteristics with three latent classes for male.

Age, as an auxiliary variable, had significant effect on the class assignment $(\mathrm{p}<0.05)$. Results show that older females are likely to be classified in Classes one and two than in Class three.

\section{Class differences in need satisfaction}

Three MANCOVA were performed to assess whether latent profile differed on motivation behavioral regulation and needs satisfaction.

Male - age was entered as covariate, significant multivariate profile effect was found, $[F(2,894)=75.37$, $\mathrm{p}<0.01$, Pillai's Trace, $\left.\eta^{2}=0.43\right]$ on external regulation, $\left(F=142.02, \quad \mathrm{p}<0.01, \eta^{2}=0.24\right)$, introjected $(F=170.60$, $\left.\mathrm{p}<0.01, \quad \eta^{2}=0.28\right), \quad$ identified $\quad(F=455.49, \quad \mathrm{p}<0.01$, $\left.\eta^{2}=0.51\right)$, intrinsic motivation $(F=264.03, \quad \mathrm{p}<0.01$, $\left.\eta^{2}=0.37\right)$, amotivation $\left(F=470.26, \mathrm{p}<0.01, \eta^{2}=0.51\right)$, competence $\left(F=112.30, \mathrm{p}<0.01, \eta^{2}=0.20\right)$, autonomy $\left(F=96.86, \quad \mathrm{p}<0.01, \quad \eta^{2}=0.18\right)$, relatedness $(F=128.45$, $\left.\mathrm{p}<0.01, \eta^{2}=0.22\right)$. Age is not significant as covariate ( $p>0.05)$. Post-hoc tests showed that self-determined group reported higher levels of perceived competence than those in moderately self-determined motivated and amotivated groups $(\mathrm{p}<0.01$, Table 4$)$. The differences between the moderately self-determined motivated and amotivated groups are not significant in perceived autonomy and perceived relatedness ( $p>0.01$ ).

Female - significant profile effect was found $\left[F(2,986)=81.58, \mathrm{p}<0.01\right.$, Pillai's Trace, $\left.\eta^{2}=0.43\right)$ on external regulation $\left(F=37.44, \mathrm{p}<0.01, \eta^{2}=0.07\right)$, introjected $\left(F=556.33, \quad \mathrm{p}<0.01, \quad \eta^{2}=0.53\right)$, identified $(F=1182.08$, $\left.\mathrm{p}<0.01, \eta^{2}=0.71\right)$, intrinsic motivation $(F=316.00, \mathrm{p}<0.01$, $\left.\eta^{2}=0.39\right)$, amotivation $\left(F=88.38, \mathrm{p}<0.01, \quad \eta^{2}=0.15\right)$, competence $\left(F=134.20, \mathrm{p}<0.01, \eta^{2}=0.21\right)$, autonomy $\left(F=88.98, \quad \mathrm{p}<0.01, \quad \eta^{2}=0.15\right)$, relatedness $(F=189.38$,

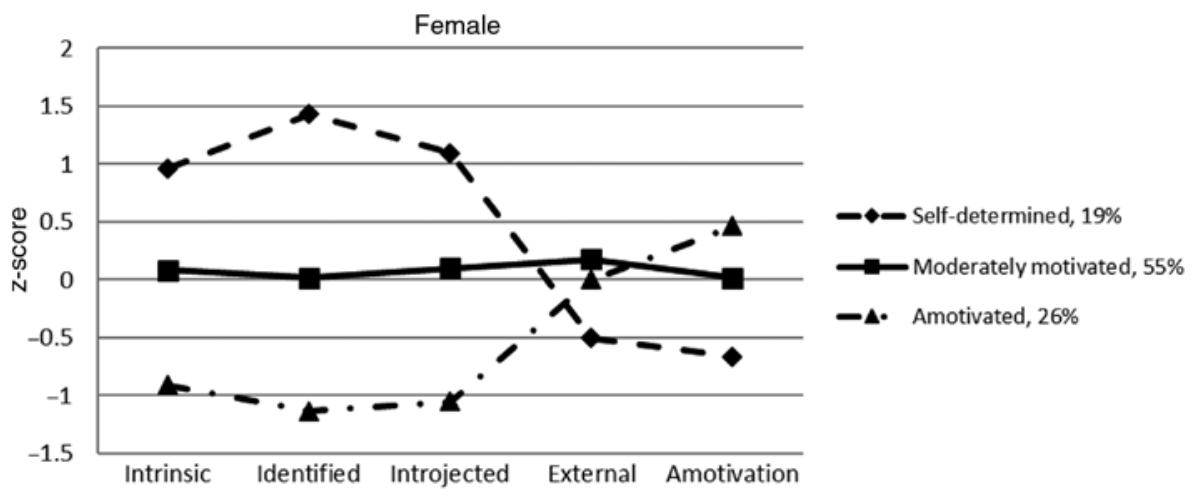

Figure 2: Profile characteristics with three latent classes for female. 
$\left.\mathrm{p}<0.01, \eta^{2}=0.28\right)$. Age is not significant as covariate $(p>0.05)$. Post-hoc tests showed that self-determined group reported highest levels of all outcome variables $(p<0.01$, Table 4). The other two groups (i.e. amotivated and intrinsic and extrinsic motivated groups) did not differ in terms of all three types of needs satisfaction ( $p>0.01)$. In general, regardless of gender, the magnitude of all profile differences was large (above 0.14) [52].

\section{Discussion}

The purpose of the present study was to assess the motivational profile in both Chinese male and female university students. The three-cluster solution was aligned with prior findings in academic [32] and physical education contexts [33,35]. The overall patterns of the motivational profiles for both genders were similar. The majority of students belong to moderately motivated profile. In this profile, students scored average in all forms of motivation regulations, except amotivation. In the second profile (self-determined profile), students scored high levels in self-determined motivation (i.e. intrinsic motivation and identified regulation), moderate levels of introjected regulation and low levels of external regulation and amotivation. Students with self-determined motivation profile had higher values on three types of needs satisfaction. Consistent with the theoretical tenet of the SDT [9] and empirical findings, the satisfaction of the three psychological needs of motivation was a positive predictor to higher selfdetermined behavioral intention in physical activity [38]. Furthermore, the current findings found that males (44\%) were more likely than their female counterparts (19\%) in self-determined profile. This further supports the qualitative difference of motivational profiles of participants in physical activity contexts [28, 35].

Consistent with prior profile research $[26,35,36]$, an amotivated group (non self-motivated group) was found in both male and female groups. In this profile, students scored lowest in all needs (i.e. competence, autonomy, relatedness). Students from the amotivated group experienced lower satisfaction in all three needs of the SDT. This is worrisome, especially as $26 \%$ of females belong to this profile. This is consistent with a prior study, in which male Hong Kong Chinese college students reported a higher level of physical activity than their female counterparts [22]. These findings deserve our attention as previous research showed an inverse relationship between amotivation and effort in physical education [57], but no association with intention to exercise $[58,59]$. 
The present study extends the literature about the importance of understanding the qualitatively different reason to participate in a target behavior. "This way of identifying homogenous groups may be more realistic than artificially imposing a structure on the observed data" [36]. Most previous work had not examined gender differences based on the mean score of each motivational regulation, except one academic study [24]. Not much published physical activity research explored the motivational profiles in separate groups (gender: male and female). The present study extended prior studies by using a person-centered approach to explore the motivational regulation profiles among the Chinese university students and to compare gender differences on the pattern of motivation profiles and its effects on SDT correlates (needs satisfaction).

The gender difference was found in the non self-motivated profile. The current study supported the proposition of the co-occurrence of the two distinct motivations. By using this person-oriented approach, students reported the optimal level of needs satisfaction when endorsing both high intrinsic (intrinsic and identified regulation) and moderate extrinsic motivations (introjected regulation). This was further supported by [60] who found that self-determined motivational profile (higher intrinsic motivation, identified regulation, introjected regulation, and lower external regulation) was positively associated with perceptions of competence, autonomy and relatedness. This was consistent with academic research that was conducted among high school [32] and college students [61]. The present study extended SDT research, which was dominated by research using the variable-oriented approach and showed co-existence of both intrinsic and extrinsic motivations.

From a practical view, educators or practitioners should foster students' internalization and develop selfdetermined forms of behavioral regulations by establishing an autonomy-supportive learning atmosphere to motivate students to engage in physical activity, such as allowing them to participate in decision-making, providing an optimal achievable task, introducing peer learning group, building relationships with others, and improving their understanding of the importance of physical activity, thereby promoting self-determined motivation toward physical activity. Researchers highlighted the role of culture in producing uniquely distinct SDT motivational profiles [27]. More work should be replicated in different cultural contexts to assess the pattern of motivational profile and its impact on SDT correlates. For example, Moran and colleagues [27] identified a five-cluster solution of SDT profiles based on a sample of Chinese employees.
However, it might be context-specific. Therefore, clearly, more research is warranted to increase the generalizability of the motivational research in physical activity contexts.

Several limitations were noted in the present study. First, the cross-sectional nature of the research design might limit the findings of the study. Future work might examine the temporal stability of these motivational profiles across time [62]. Second, only antecedents were included. More studies are warranted to explore how the relationships between motivational regulations and its affective and behavioral outcomes would differ by gender. It is possible that behavioral outcomes, such as enjoyment, effort or level of physical activity, may differ among motivational profiles. Discriminant function analysis, [60] found that self-determined motivation predicted positive changes in exercise participation over time for both boys and girls. In particular, introjected regulation is a significant predictor to exercise behavior for boys, but not for girls. Lastly, more research is needed to see whether the present findings could be replicated in other non-western contexts, in which autonomy is less valued compared to western cultures [63].

Acknowledgments: This study was supported by the Startup Fund for New Recruits from the Hong Kong Polytechnic University (account code: 1-ZE36).

\section{References}

1. American College Health Association. Healthy campus 2010: make it happen 2002. URL: https://www.acha.org/healthycampus.

2. Healthy people 2010: understanding and improving health. Washington, DC: US Dept. of Health and Human Services, 2000.

3. Melton BF, Bigham LE, Bland HW, Bird M, Fairman C. Healthrelated behaviors and technology usage among college students. Am J Health Behav 2014;38:510-8.

4. Deci EL, Ryan RM. Intrinsic motivation and self-determination in human behavior. New York: Plenum, 1985.

5. Deci EL, Ryan RM. The "what" and "why" of goal pursuits: human needs and the self-determination of behavior. Psychol Inq 2000;11:227-68.

6. Edmunds J, Ntoumanis N, Duda JL. A test of self-determination theory in the exercise domain. J Appl Soc Psychol 2006;36: 2240-65.

7. Hagger M, Chatzisarantis N. Self-determination theory and the psychology of exercise. Int Rev Sport Exerc Psychol 2008;1: 79-103.

8. Mullan E, Markland D, Ingledew DK. A graded conceptualisation of self-determination in the regulation of exercise behaviour: development of a measure using confirmatory factor analytic procedures. Pers Individ Dif 1997;23:745-52.

9. Deci EL, Ryan RM. Handbook of self-determination research. Rochester, NY: University of Rochester Press, 2002. 
10. Koestner R, Losier GG. Distinguishing three ways of being internally motivated: a closer look at introjection, identification, and intrinsic motivation. In: Deci EL, Ryan RM, editors. Handbook of self-determination research. Rochester, NY: University of Rochester Press, 2002:101-21.

11. Owen KB, Astell-Burt T, Lonsdale C. The relationship between self-determined motivation and physical activity in adolescent boys. J Adolesc Health 2013;53:420-2.

12. Sabiston CM, Brunet J, Kowalski KC, Wilson PM, Mack DE, Crocker PR. The role of body-related self-conscious emotions in motivating women's physical activity. J Sport Exerc Psychol 2010;32:417-37.

13. Barbeau A, Sweet SN, Fortier M. A path-analytic model of self-determination theory in a physical activity context. J Appl Biobehav Res 2009;14:103-18.

14. Standage M, Sebire S, Loney T. Engagement in objectively assessed bouts of moderate-intensity exercise: a self-determination theory perspective. J Sport Exerc Psychol 2008;30:337-52.

15. Teixeira PJ, Carraça EV, Markland D, Silva MN, Ryan RM. Exercise, physical activity, and self-determination theory: a systematic review. Int J Behav Nutr Phys Act 2012;9:78-108.

16. Duda JL, Allison MT. Cross-cultural analysis in exercise and sport psychology: a void in the field. J Sport Exerc Psychol 1990;12:114-31.

17. Duda J, Hayashi C, Duda J. Measurement issues in cross-cultural research within sport and exercise psychology. Adv Sport Exerc Psychol Meas 1998;7:471-83.

18. Lonsdale C, Sabiston CM, Taylor IM, Ntoumanis N. Measuring student motivation for physical education: examining the psychometric properties of the perceived locus of causality questionnaire and the situational motivation scale. Psychol Sport Exerc 2011;12:284-92.

19. Deci EL, Koestner R, Ryan RM. A meta-analytic review of experiments examining the effects of extrinsic rewards on intrinsic motivation. Psychol Bull 1999;125:627-68.

20. Iyengar SS, Lepper MR. Rethinking the value of choice: $a$ cultural perspective on intrinsic motivation. J Pers Soc Psychol 1999;76:349-66.

21. Martin SB, Morrow JR, Jackson AW, Dunn AL. Variables related to meeting the CDC/ACSM physical activity guidelines. Med Sci Sports Exerc 2000;32:2087-92.

22. Lee RL, Loke AJ. Health-promoting behaviors and psychosocial well-being of university students in hong kong. Public Health Nurs 2005;22:209-20.

23. Mok WK, Lee AY. A case study on application of the theory of planned behaviour: predicting physical activity of adolescents in hong kong. J Commun Med Health Educ 2013;3:231-7.

24. Vallerand RJ, Fortier MS, Guay F. Self-determination and persistence in a real-life setting: toward a motivational model of high school dropout. J Pers Soc Psychol 1997;72:1161-76.

25. Standage M, Duda JL, Ntoumanis N. Students' motivational processes and their relationship to teacher ratings in school physical education: a self-determination theory approach. Res Q Exerc Sport 2006;77:100-10.

26. Wang C, Biddle S. Young people's motivational profiles in physical activity: a cluster analysis. J Sport Exerc Psychol 2001;23:1-22.

27. Moran CM, Diefendorff JM, Kim T-Y, Liu Z-Q. A profile approach to self-determination theory motivations at work. J Vocat Behav 2012;81:354-63.
28. Ullrich-French S, Cox A. Using cluster analysis to examine the combinations of motivation regulations of physical education students. J Sport Exerc Psychol 2009;31:358-79.

29. Lim BS, Wang CK. Perceived autonomy support, behavioural regulations in physical education and physical activity intention. Psychol Sport Exerc 2009;10:52-60.

30. Wang C, Chatzisarantis NL, Spray CM, Biddle SJ. Achievement goal profiles in school physical education: differences in selfdetermination, sport ability beliefs, and physical activity. $\mathrm{Br}$ J Educ Psychol 2002;72:433-45.

31. Marsh H, Lüdtke 0 , Trautwein U, Morin AS. Classical latent profile analysis of academic self-concept dimensions: synergy of person- and variable-centered approaches to theoretical models of self-concept. Struct Equ Modeling 2009;16:191-225.

32. Ratelle CF, Guay F, Vallerand RJ, Larose S, Senécal C. Autonomous, controlled, and amotivated types of academic motivation: a person-oriented analysis. J Educ Psychol 2007;99:734-46.

33. Boiché J, Sarrazin PG, Grouzet FM, Pelletier LG, Chanal JP. Students' motivational profiles and achievement outcomes in physical education: a self-determination perspective. J Educ Psychol 2008;100:688.

34. Cox AE, Ullrich-French S, Sabiston CM. Using motivation regulations in a person-centered approach to examine the link between social physique anxiety in physical education and physical activity-related outcomes in adolescents. Psychol Sport Exerc 2013;14:461-7.

35. Ntoumanis N. Motivational clusters in a sample of british physical education classes. Psychol Sport Exerc 2002;3:177-94.

36. McNeill MC, Wang CK. Psychological profiles of elite school sports players in Singapore. Psychol Sport Exerc 2005;6:117-28.

37. Markland D, Tobin V. A modification to the behavioural regulation in exercise questionnaire to include an assessment of amotivation. J Sport Exerc Psychol 2004;26:191-6.

38. Wilson PM, Rodgers WM. The relationship between perceived autonomy support, exercise regulations and behavioral intentions in women. Psychol Sport Exerc 2004;5:229-42.

39. Ingledew DK, Markland D. Three levels of exercise motivation. Appl Psychol Health Well Being 2009;1:336-55.

40. Vlachopoulos SP, Kaperoni M, Moustaka FC. The relationship of self-determination theory variables to exercise identity. Psychol Sport Exerc 2011;12:265-72.

41. Markland D, Tobin VJ. Need support and behavioural regulations for exercise among exercise referral scheme clients: the mediating role of psychological need satisfaction. Psychol Sport Exerc 2010;11:91-9.

42. Wilson PM, Rogers WT, Rodgers WM, Wild TC. The psychological need satisfaction in exercise scale. J Sport Exerc Psychol 2006;28:231-51.

43. Gunnell KE, Wilson PM, Zumbo BD, Mack DE, Crocker PR. Assessing psychological need satisfaction in exercise contexts: Issues of score invariance, item modification, and context. Meas Phys Educ Exerc Sci 2012;16:219-36.

44. Gunnell KE, Crocker PR, Mack DE, Wilson PM, Zumbo BD. Goal contents, motivation, psychological need satisfaction, wellbeing and physical activity: a test of self-determination theory over 6 months. Psychol Sport Exerc 2014;15:19-29.

45. Brislin RW. The wording and translation of research instruments. In: Lonner W, Berrhy J, editors. Field methods in cross-cultural research. Beverly Hills, CA: Sage, 1986:137-64. 
46. Collins LM, Lanza ST. Latent class and latent transition analysis : with applications in the social behavioral, and health sciences. Hoboken, N): Wiley, 2010.

47. Nylund KL, Asparouhov T, Muthén BO. Deciding on the number of classes in latent class analysis and growth mixture modeling: a monte carlo simulation study. Struct Equ Modeling 2007;14:535-69.

48. Wang J, Wang X. Mixture modeling. In: Balding DJ, editor. Structural equation modeling: applications using mplus. New York: Wiley, 2012:289-390.

49. Aldridge AA, Roesch SC. Developing coping typologies of minority adolescents: A latent profile analysis. J Adolescence 2008;31:499-517.

50. Enders CK. A note on the use of missing auxiliary variables in full information maximum likelihood-based structural equation models. Struct Equ Modeling 2008;15:434-48.

51. Cohen J. Statistical power analysis for the behavioral sciences, 2nd ed. Hillsdale, NJ: Lawrence Erlbaum, 1988.

52. Curran PJ, West SG, Finch JF. The robustness of test statistics to nonnormality and specification error in confirmatory factor analysis. Psychol Methods 1996;1:16-29.

53. Hu Lt, Bentler PM. Cutoff criteria for fit indexes in covariance structure analysis: conventional criteria versus new alternatives. Struct Equ Modeling 1999;6:1-55.

54. Kline P. An easy guide to factor analysis. London: Routledge, 1994.

55. Browne MW, Cudeck R. Alternative ways of assessing model fit. In: Bollen KA, Long JS, editors. Testing structural equation models. Newbury Park, CA: Sage, 1993:136-62.
56. Byrne BM. Structural equation modeling with AMOS : basic concepts, applications, and programming. Mahwah, NJ: Lawrence Erlbaum, 2001.

57. Shen B, Wingert RK, Li W, Sun H, Rukavina PB. An amotivation model in physical education. J Teach Phys Educ 2010;29: 72-84.

58. Standage M, Duda JL, Ntoumanis N. A model of contextual motivation in physical education: using constructs from selfdetermination and achievement goal theories to predict physical activity intentions. J Educ Psychol 2003;95:97-110.

59. Taylor IM, Ntoumanis N, Standage M, Spray CM. Motivational predictors of physical education students' effort, exercise intentions, and leisure-time physical activity: a multilevel linear growth analysis. J Sport Exerc Psychol 2010;32:99-120.

60. Gillison FB, Standage M, Skevington SM. Motivation and bodyrelated factors as discriminators of change in adolescents' exercise behavior profiles. J Adolesc Health 2011;48:44-51.

61. Smith JL, Deemer ED, Thoman DB, Zazworsky L. Motivation under the microscope: Understanding undergraduate science students' multiple motivations for research. Motiv Emot 2014;38:496-512.

62. Hayenga A, Corpus J. Profiles of intrinsic and extrinsic motivations: A person-centered approach to motivation and achievement in middle school. Motiv Emot 2010;34:371-83.

63. Markus HR, Kitayama S. Models of agency: Sociocultural diversity in the construction of action. In: Murphy-Berman $\mathrm{V}$, Berman J, editors. Nebraska symposium on motivation: crosscultural differences in perspectives on self. Lincoln, NE: University of Nebraska Press, 2003:1-57. 